\title{
Observations of a soft X-ray rising loop associated with a type II burst and a coronal mass ejection in the 03 November 2003 X-ray flare
}

\author{
C. Dauphin ${ }^{1}$, N. Vilmer ${ }^{1}$, and S. Krucker ${ }^{2}$ \\ 1 LESIA, Observatoire de Paris, 92195 Meudon Cedex, France \\ e-mail: cyril. dauphin@obspm. fr \\ 2 Space Sciences Laboratory, University of California, Berkeley, CA-94720, USA \\ Received 17 November 2005 / Accepted 13 April 2006
}

ABSTRACT

\begin{abstract}
Context. We report observations of a type II burst - the signature of a shock wave - starting at the unusual high frequency of $650 \mathrm{MHz}$ during the 03 November 2003 flare. This flare is associated with the propagation of a soft X-ray coronal loop and with a coronal mass ejection (CME).

Aims. We study in this paper the origin of the shock wave in the low corona and present a kinematics analysis of the soft X-ray coronal loop and of the CME observed a few tens of minutes later.

Methods. We study in this paper the spatial and temporal relation between the soft X-ray rising loop observed by the GOES soft X-ray Imager (GOES/SXI), the type II sources observed by the Nançay Radio Heliograph (NRH) and the CME observed by LASCO (Large Angle and Spectroscopic Coronograph).

Results. This analysis shows that the type II burst observed during this flare is driven by the X-ray rising loop. Furthermore, the kinematics analysis of the X-ray coronal loop and CME shows that the two structures are related.

Conclusions. The direct comparison of the type II sources with the GOES/SXI observations clearly shows that the type II burst is ignited by the shock wave created ahead of the rising X-ray loop. Finally, we propose to interpret these different observations in the framework of an ascending magnetic structure with a reconnecting process operating in the tail of this ascending structure.
\end{abstract}

Key words. Sun: flares - Sun: radio radiation - Sun: coronal mass ejections (CMEs)

\section{Introduction}

Type II bursts at decimetric to dekametric wavelengths (Nelson \& Melrose 1985) are believed to be the indirect radio signatures of large scale MHD shock waves propagating through the solar corona (see e.g. Nelson \& Melrose 1985; Uchida 1960; Wild 1962; Uchida 1974). Although this scenario of shock accelerated electrons exciting plasma waves converted into escaping radio waves is generally accepted, the origin of the shock wave is still not clear. Two origins have been proposed for the production of the shock wave:

- a blast wave initiated by e.g. a pressure pulse or a short localized energy release in a flare (e.g. Vrsnak \& Lulic 2000). In that case, no mass motion drives the shock wave.

- the propagation of coronal material (piston-driven scenario) at super-Alfvenic speeds (e.g. Maxwell et al. 1985). A plasmoid ejection or a coronal mass ejection (CME) may constitute the piston and creates a shock wave ahead.

The first scenario is supported by the observations that type II bursts in the low and middle corona (i.e. at metric wavelengths) are usually associated in time with flares which initiate in their impulsive phase many waves (see e.g. Vrsnak et al. 2005). Historically, large scale disturbances propagating in the chromosphere at speed around $1000 \mathrm{~km} \mathrm{~s}^{-1}$ (Moreton waves) have been first observed at $\mathrm{H} \alpha$ wavelengths (Moreton 1960). In the last decade, EUV observations from the Extreme Ultraviolet Telescope (EIT) on SOHO (Delaboudinière et al. 1995) have also shown global wave like features (Thompson et al. 1998) propagating in the corona with smaller speeds around $250 \mathrm{~km} \mathrm{~s}^{-1}$ (EIT waves). More recently, global wave signatures have been discovered in the X-ray domain with Yohkoh (e.g. Hudson et al. 2003) and GOES soft X-ray imager (SXI) (Warmuth et al. 2005) and at radio wavelengths with the Nançay Radio Heliograph (NRH; Kerdraon \& Delouis 1997) (Vrsnak et al. 2005). Although there is no doubt that waves are present during flares, the link between these different waves and the link between these waves and the type II bursts are still under debate. Regarding the second question, few combined observations have been reported in the literature supporting the blast wave origin of the metric type II shock. Klassen et al. (2000) have shown that $90 \%$ of type II bursts (sample of 20 events) are associated to EIT waves but that there is some discrepancy between the speeds derived from type II bursts and from EIT waves. While Gopalswamy et al. (2000) reported spatial association between an EIT wave and the localization of a metric type II burst, Khan \& Aurass (2002) reported a close temporal and spatial association between a soft X-ray wave and the source location of the type II burst. In this case, extrapolations of the leading edge of the propagating soft X-ray disturbance show a close association with both the Moreton and EIT waves observed in the same flare, therefore suggesting the common origin of all these waves. Such an observation supports the blast wave scenario for the origin of the metric type II burst as no mass motion nor CME were detected during this event. However, as pointed out by Cliver et al. (2005), the detection of coronal mass ejections is difficult 
for flares close to the disk center such as the one studied by Khan \& Aurass (2002). Furthermore, the detection of coronal mass ejections close to the solar surface is rare and based on the few observations combining the Large Angle and Spectroscopic Coronagraph (LASCO, Brueckner et al. 1995) C1 and C2 and EIT (see e.g. Zhang et al. 2001) and of some observations of EIT rising low coronal loops or prominence eruptions seen at the limb (see e.g. Vilmer et al. 2003; Cremades \& Bothmer 2004).

The alternative scenario which postulates that coronal shocks leading to type II bursts are driven by mass motions: ejecta, CMEs or their counterparts close to the solar disk needs to be investigated by analysing combined images of ejecta and CMEs and type II radio sources at similar coronal heights.

At high altitude in the corona as well as in the interplanetary medium, the lack of radio heliograph observations makes impossible the direct comparison of the type II source locations with CME observations. Correlative studies between type II bursts and CMEs have therefore been made using the CME dynamics inferred from the Large Angle and Spectroscopic Coronagraph on SOHO (LASCO) and the shock dynamics inferred from radio spectrographs. According to these studies, dekametric type II bursts are found to be related to CMEs (Reiner et al. 2001; Gopalswamy et al. 2001). Although no radio images are available for the dekametric type II bursts, the sources are expected to be in front of the CME.

At lower altitude in the corona, poor correlations are found between metric type IIs bursts and CMEs. The apparent lack of relationship between speeds (e.g. Reiner et al. 2001), starting times or extrapolated spatial positions apparently deserve the piston-driven scenario. However, as pointed out by Mancuso \& Raymond (2004), the type II height depends on the coronal density model used and on geometrical effects. Moreover, the onset time of CMEs is usually derived from coronagraph data, which are limited to observations above an altitude where CMEs are often no longer accelerated as was shown by Zhang et al. (2001) using combined EIT and LASCO C1, C2 data to determine the launch time without any extrapolation. These combined observations indeed show a three phase evolution with a slow rise phase followed by an impulsive acceleration and, in fine, a last phase of quasi-constant velocity. By using data from the Transition and Coronal Explorer TRACE (Handy et al. 1999), the SOHO Ultraviolet Coronal Spectrometer UVCS (Kohl et al. 1995) and LASCO, Gallagher et al. (2003) have studied the CME kinematics at low altitudes and shown that an exponential rise was required to reproduce the observed height time evolution. The acceleration and velocity values are similar to the ones found by Alexander et al. (2002) combining YOHKOH/SXT (Soft X-ray Telescope) and LASCO data for other events. These different observations have led to the conclusion that the CME acceleration is associated to the impulsive phase of the flare. This also implies that the real onset time of a CME differs from the one found from the simple extrapolation of the LASCO C2 and C3 data points. Is is also expected that CME produces waves during the fast acceleration phase which may explain the correlation found between global waves and flares. Cliver et al. (2005) have finally clearly demonstrated by reanalysing several events already published that the CME/metric type II timing problem can be solved when considering that the CME is accelerated during the impulsive phase of the flare. As a consequence, the piston-driven scenario can be reconsidered for metric type II bursts.

In support of a driven shock wave even at low altitudes, Gopalswamy et al. (1997) reported observations of a rapidly rising X-ray structure in association with the type II burst onset and Klein et al. (1999) have shown a clear example of a metric type II burst with a candidate flare ejecta driver observed at the same heights in the corona. On the other hand, Klassen et al. (1999) reported that among five events analysed in only one event the onset of type II emission was associated with evolving X-ray loops. This limited number of events in which X-ray ejecta and metric type II bursts have been associated can be explained by the fact that the temperature or density of the plasma compressed by the shock are too low to be detectable by X-ray observations and by the further difficulty of detecting X-ray ejecta for flares close to the disk center. Limb observations are probably the best way to clearly detect mass motions in the corona. Thus, more direct observations of type II sources in the low corona and of CMEs at low altitude are needed to progress on this topic.

We present in this paper observations of the X-ray rising loops observed by the GOES Soft X-ray Imager (GOES/SXI, Hill et al. 2005) during the 3 November 2003 GOES X3.9 flare at $\sim 99: 45$ UT which occurred in NOAA active region 0488 (Liu et al. 2004; Veronig et al. 2006). We shall focus in this paper on the kinematics analysis of the X-ray loops and of the LASCO CME observed in association with this flare. We also compare the position of the X-ray rising loops to the location of the type II radio sources observed with the $\mathrm{NRH}$ at the same altitude. In the usual framework for CMEs, while a shock wave possibly producing the type II emission is developed ahead of the erupting flux rope, reconnection may occur below, possibly producing new episodes of particle acceleration. The peculiarity of the 03 November 2003 flare is the presence of two phases of intense emissions in the hard X-ray and radio domains separated in time by a few minutes. Such events are rare and present variable delays between the two phases (see the example in Asai et al. 2001). We shall investigate whether the later phase can be a signature of some reconnection below the flux rope as was suggested for another event by Pick et al. (2005).

The paper is organised as follows: Sect. 2 gives the observational background of the flare. In Sect. 3, we describe the GOES/SXI observations, the relationship between the X-ray rising loops, the $\mathrm{CME}$ and the metric/decimetric radio emissions. The relationship between X-ray rising loops and the onset of type II emission is presented in Sect. 4. A summary of the main observational results from this paper and a previous paper (Dauphin et al. 2005) are discussed in Sect. 5. A potential flare/CME scenario based on these observations is presented and the fact that the X-ray rising loop may be the driver of the decimetric type II burst is emphasized.

\section{General description of the event and origin of the continuum emission after 09:57:00 UT}

The 03 November 2003 event at $\sim 09: 45$ UT is one of the series of large X-ray flares between October 28 and November 04, 2003. This flare is not among the most energetic ones of the series but it has been observed by many instruments and in a wide range of wavelengths. It also shows an unusual energetic late phase. Figure 1 shows the time evolution of the GOES X-ray flux, together with the RHESSI counts (background not subtracted) in the 100-150 keV hard X-ray energy band and the radio flux observed at $610 \mathrm{MHz}$ from the TRIESTE observatory and at $432 \mathrm{MHz}, 410 \mathrm{MHz}, 327 \mathrm{MHz}, 236 \mathrm{MHz}$ and $164 \mathrm{MHz}$ with the Nancay Radio Heliograph. The two bottom plots represent a composite radio spectrum from PHOENIX-2 in the $2 \mathrm{GHz}-400 \mathrm{MHz}$ range, and OSRA (Potsdam) from $400 \mathrm{MHz}$ to $40 \mathrm{MHz}$. 


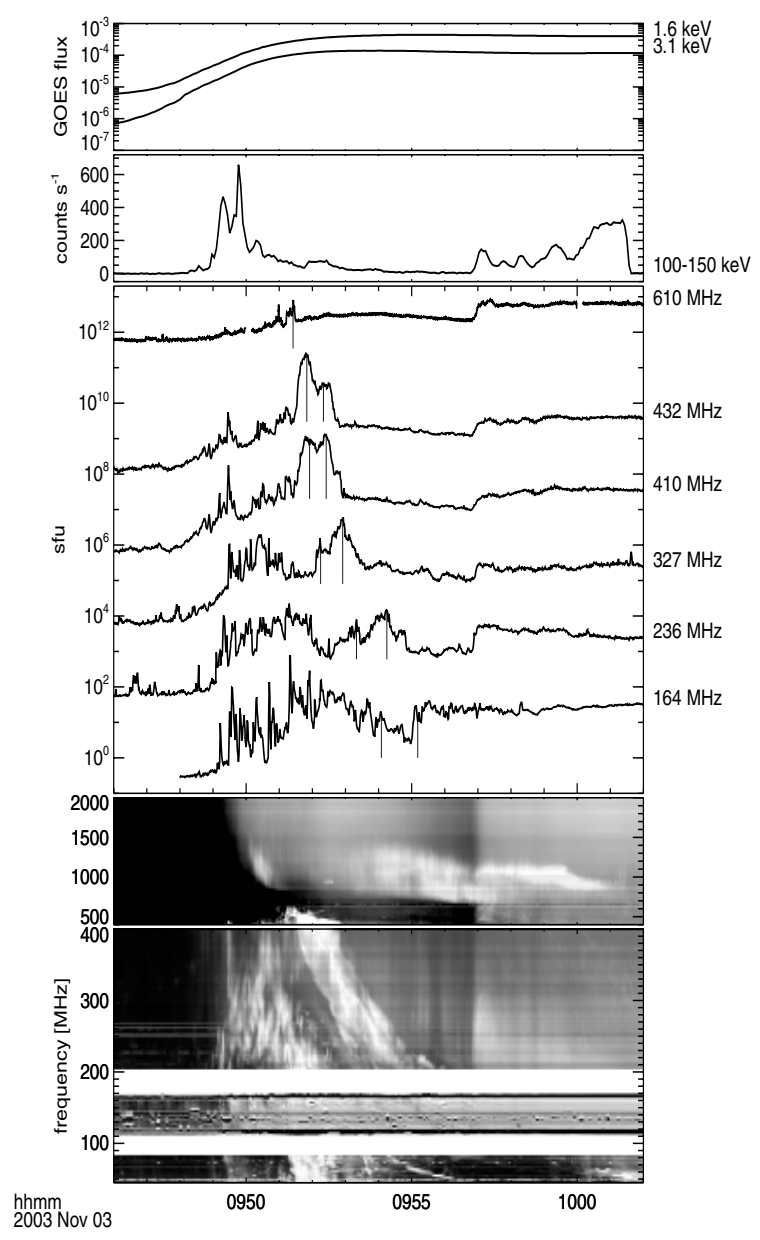

Fig. 1. From top to bottom: time evolution of the GOES X-ray flux (panel 1) and of the X-ray RHESSI counts in the 100-150 keV energy band (background not subtracted) (panel 2); time evolution of the radio flux observed at $610 \mathrm{MHz}$ (from the TRIESTE observatory), $432 \mathrm{MHz}$, $410 \mathrm{MHz}, 327 \mathrm{MHz}, 236 \mathrm{MHz}$ and $164 \mathrm{MHz}$ (from the Nancay radioheliograph). Radio composite spectrum observed between $2 \mathrm{GHz}$ to $1 \mathrm{MHz}$ by PHOENIX-2 (ETH Zürich) (panel 3), OSRA (AIP Potsdam) (panel 4) (the time is in UT). Note the continuum enhancement at 09:57 UT corresponding to the second phase of energy release observed in hard X-ray wavelength range.

At energies above $100 \mathrm{keV}$ hard X-ray emission presents two broad series of bursts around, respectively, 09:50 UT and after 09:57 UT. These two parts observed up to $\sim 450 \mathrm{keV}$ are separated by a period in which only X-ray emission below $100 \mathrm{keV}$ is observed. While the first part of the X-ray emission above $100 \mathrm{keV}$ is associated in the $\mathrm{dm} / \mathrm{m}$ domain to type III like bursts, the second part after 09:57 UT is, apart from narrow band slowly drifting emissions around $800 \mathrm{MHz}$, mainly associated with a strong continuum emission in the whole range from $2 \mathrm{GHz}$ to almost $200 \mathrm{MHz}$. Furthermore, modulations of this strong continuum emission are observed in phase in the whole energy band going from $89 \mathrm{GHz}$ to $327 \mathrm{MHz}$ and extend to $164 \mathrm{MHz}$ for the fourth and fifth modulations at 09:59 UT and 10:00 UT. These modulations follow similar modulations in the hard X-ray domain and have been discussed more extensively in Dauphin et al. (2005).

Decimetric/metric imaging observations of this event have been described in Dauphin et al. (2005). At the time of type III like bursts in the decimetric domain (i.e. 09:49:00 UT to 09:51:00 UT) radio emission comes primarily from three sources: one close to the limb and to the active region and two others above the limb associated with the bursts. During the first part of the flare, the positions of bursts at different frequencies show some spatial dispersion with frequency as expected for type III like bursts. During the modulations however, radio emitting sources at different frequencies tend to be more co spatial (see Dauphin et al. 2005, for details). During all the modulations, radio emission originates from extended sources which consist at high frequencies (432 MHz to $327 \mathrm{MHz}$ ) of three sources with varying intensities. After the first modulations, radio emitting sources in the low frequency range (i.e. $164 \mathrm{MHz}$ to $236 \mathrm{MHz}$ ) show extension to the north as also described in Vrsnak et al. (2005).

Dauphin et al. (2005) have also performed a spectral analysis and shown that gyrosynchrotron emission is the prevailing emission mechanism even at decimetric wavelengths for the broad-band radio emission. Indeed, during the modulations, the radio spectrum continuously rises from $327 \mathrm{MHz}$ to $35 \mathrm{GHz}$. The observations of gyro synchrotron emission at decimetric wavelengths during the second part of the flare could be explained by a strong decrease of the plasma density in the metric radio sources in this phase. From the observations of synchrotron emission at $327 \mathrm{MHz}$ (including the measurements of the flux and of the source size) Dauphin et al. (2005) deduced an upper limit of the density of $3 \times 10^{7} \mathrm{~cm}^{-3}$ and a magnetic field value of $2 \mathrm{G}$ (this would lead to an Alfven speed around $740 \mathrm{~km} \mathrm{~s}^{-1}$ ). The two parts of the hard X-ray flare above $100 \mathrm{keV}$ are separated by a decimetric/metric type II emission starting at $\sim 09: 51: 12$ UT. Figure 1 shows that the harmonic component of this type II starts at the unusual high frequency of $650 \mathrm{MHz}$. As already observed in other events, the start of the type II emission does not correspond to a strong production of X-ray emission at high energies (see e.g. Klein et al. $1999,2003)$. A fast coronal mass ejection $\left(1420 \mathrm{~km} \mathrm{~s}^{-1}\right)$ is observed by the LASCO coronagraph on SOHO and its extrapolated starting time derived from a height-time analysis from a linear fit or a second order fit of the positions of the CME observed in C2 and C3 is between 09:53:17 UT and 09:54:35 UT (http://cdaw.gsfc.nasa.gov/CME_list/index.html).

\section{Rising X-ray source and CME onset}

\subsection{Detection of $X$-ray rising loops with GOES/SXI}

Soft X-ray images of the 03 November 2003 flare are available from the Geostationary Operational Environmental Satellites (GOES) Soft X-ray Imager (SXI) (Hill et al. 2005) which provides images in three different exposition modes (without and with filters) allowing a rudimentary plasma temperature discrimination. Without filter, GOES/SXI system offers an excellent response for a coronal temperature between 1 and $4 \mathrm{MK}$ with a peak at 3.4 MK. The denser filters A and B shift and narrow the response to higher temperature with a peak at, respectively, 3.8 MK and 4.0 MK. Figure 2 shows GOES/SXI raw images obtained without filter at 09:46:18 UT and 09:50:18 UT. The image at 09:50:18 UT shows extension of loop like features heated to $23.4 \mathrm{MK}$ observed north-west from the inner active region whose emission saturates the detector. No corresponding structure is detected at this location either in the same conditions with GOES/SXI at 09:46:18 UT, or at 09:46:22 UT in SOHO/EIT $195 \AA$, which shows coronal structures around 1.5 MK (see Fig. 2).

Difference GOES/SXI images taken in the same exposition mode show the evolution and the expansion of the loop like structure toward the north-west (Fig. 3). The time of the 

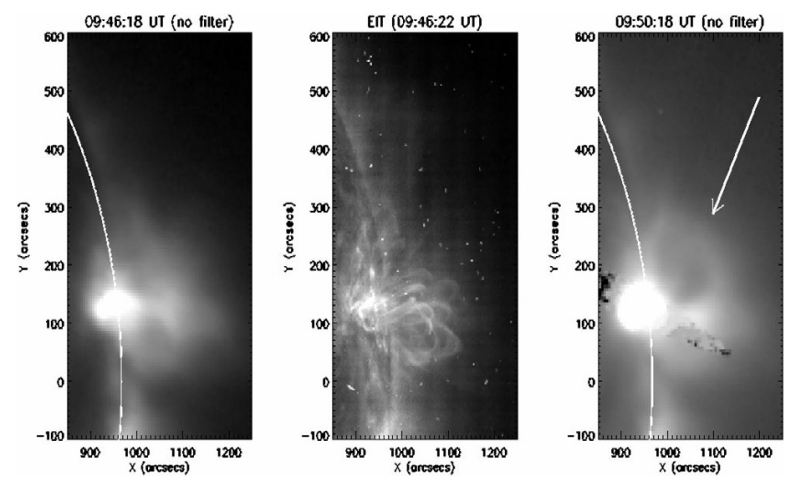

Fig. 2. GOES/SXI raw images of the 03 November 2003 flare at 09:46:18 UT and 09:50:18 UT showing the expansion of a rising loop like feature (indicated by a white arrow) in the north east direction (the white curve indicates the solar limb). The images are taken with no filter and correspond to the detection of plasma with a peak temperature of 3.4 MK. Note the saturation of the detector in connection with the flare for the image taken at 09:50:18 UT. The EIT $195 \AA$ at 09:46:22 UT shows coronal structures around 1.5 MK. There is no EIT loop corresponding before the flare to the X-ray loop observed at 09:50:18 UT.

reference image with no filter and with filter $\mathrm{A}$ is taken resp. at 10:02:17 UT and 10:00:19 UT when the propagating feature is outside the GOES/SXI field of view. For filter B, the time of the reference image is taken at 09:39:33 UT, i.e. before the flare. In these conditions, the location of the bright hot feature in the difference images is representative of the position of hot material at the time of the image which is not used as the reference image. While the first image at 09:47:33 UT either could show a hot coronal source or the start of a plasmoid ejection, the following images show clearly an expanding loop. As usually observed, expanding loops become fainter as they propagate, making them more difficult to detect. Nevertheless, the front of this expanding loop system is detectable until 09:54:18 UT.

In addition, Fig. 3 shows clearly that the X-ray loop propagates in a direction and has an extent which is consistent with the CME which is observed later in the field of view of LASCO C2. Such an observation is in agreement with previously published YOHKOH SXT observations showing expanding hot and dense loop-like structures potentially associated to the onset of CMEs (see e.g. Hudson et al. 1996). It must finally be noted that there are no EIT data at the times corresponding to the expanding loops observed in GOES/SXI and shown in Fig. 3 (i.e. from 09:47:33 UT to 09:54:18 UT). Furthermore, the difference EIT image (09:46:22 UT-09:58:20 UT) does not show any loop like feature which could correspond to the one observed with GOES/SXI.

As discussed in Hudson et al. (2003), the interpretation of the expanding X-ray feature is partly ambiguous since the moving features can either reveal the propagation of waves heating the ambient plasma or of a real movement of hot matter. However, the hot material observed at 09:50:18 UT is localized in a small portion of the north-west quadrant of the solar corona, has extension only towards the north and shows foot-points like features. This rather favors the interpretation of the expanding X-ray feature in terms of the propagation of loops filled with hot matter or of ambient plasma heated ahead of a cooler plasma ejecta (i.e. a driven wave). Indeed, a blast wave signature should have a more concentric development and should not show foot-points like features.

\subsection{Relationship between the $X$-ray rising loops and the $C M E$}

Figure 4 (left panel) shows the relative position of the rising loops detected by GOES/SXI (difference image at 09:52:20 UT) and of the CME detected by LASCO. Even if the two experiments do not probe plasma at the same temperature, this figure clearly shows that the axis (indicated by a dashed line) and the shape of the X-ray rising loop could be related to the ones of the CME. This relationship also appears when considering the angular width of the X-ray loop, which can be extrapolated to the angular width of the CME. The next step to confirm the link between the X-ray loop and the CME is to study the kinematics of both structures. This is done by measuring the projected height of the front of the X-ray rising loop and of the CME along the dashed line indicated in Fig. 4. These data points, taken along the axis of the CME, are indicated in the Fig. 3 by cross marks.

Figure 5 shows the projected height as a function of time and the deduced velocity and acceleration of the front of the rising expanding loops seen in GOES/SXI and of the CME seen with LASCO C2. The position of the X-ray front seen in GOES/SXI at 09:47:33 UT, 09:50:18 UT and 09:52:20 UT is assumed to be accurate within 10 arcsec. At 09:54:18 UT the error on the position is estimated to be around 40 arcsec as the detection of the front is more difficult. The detection of the CME front seen in LASCO is assumed to be accurate within an error of 2 pixel $(1$ pixel $=11.9$ arcsec $)$. Two different models are used in the following to reproduce the observed evolution with time of the height of the GOES/SXI loop front with time: exponential time dependant acceleration (e.g. Gallagher et al. 2003) and constant acceleration (e.g. Alexander et al. 2002). In the present case, the best fit (represented by the black curve in Fig. 5) is obtained in the case of a constant acceleration resulting in the following height time profile of the GOES/SXI loop front:

$h(t)=h_{0}+V_{0} t+\frac{1}{2} a_{0} t^{2}$

where the initial condition $h_{0}$ is the height of the X-ray disturbance taken at 09:47:33 UT, $v_{0}$ and $a_{0}$ are respectively the velocity at 09:47:33 UT and the constant acceleration deduced from the fit found to be $v_{0}=31 \mathrm{~km} \mathrm{~s}^{-1}$ and $a_{0}=2746 \mathrm{~m} \mathrm{~s}^{-1}$.

The extrapolation of the height of the X-ray loop at the time of the first LASCO CME detection (10:06:05 UT) would lead with this constant acceleration to a X-ray front at 3577 arcsec (i.e. well above the CME front). In the case of a null acceleration after the last data GOES/SXI point (dashed line in Fig. 5) the front of X-ray loop would be at 2375 arcsec. These two extrapolations represent two extreme cases of the kinematics of the $\mathrm{X}$-ray loop and the true position of the front of the X-ray loop is expected to be between 2375 arcsec and 3577 arcsec. Even if the front of the X-ray loop is not expected to overlap at the same time the front of the CME the fact that the position of the CME front at 10:06 UT lies between the two extrapolated values of the $\mathrm{X}$-ray front argue in favour of a strong relationship between the $\mathrm{X}$-ray rising loop and the CME. This relationship would moreover increase in the case of a very low acceleration of the X-ray loop between 09:54:20 UT and 10:06 UT.

Figure 5 shows that the increase of the velocity with time grows rapidly around 09:50:30 UT $\sim 40 \mathrm{~s}$ after the peak time of the impulsive hard X-ray emission part at $150-300 \mathrm{keV}$. Thus the acceleration reaches its maximum shortly after the peak time of the impulsive hard X-ray phase of the flare. This has already been observed by e.g. Gallagher et al. (2003) combining TRACE and RHESSI (Lin et al. 2003) observations. Besides, 

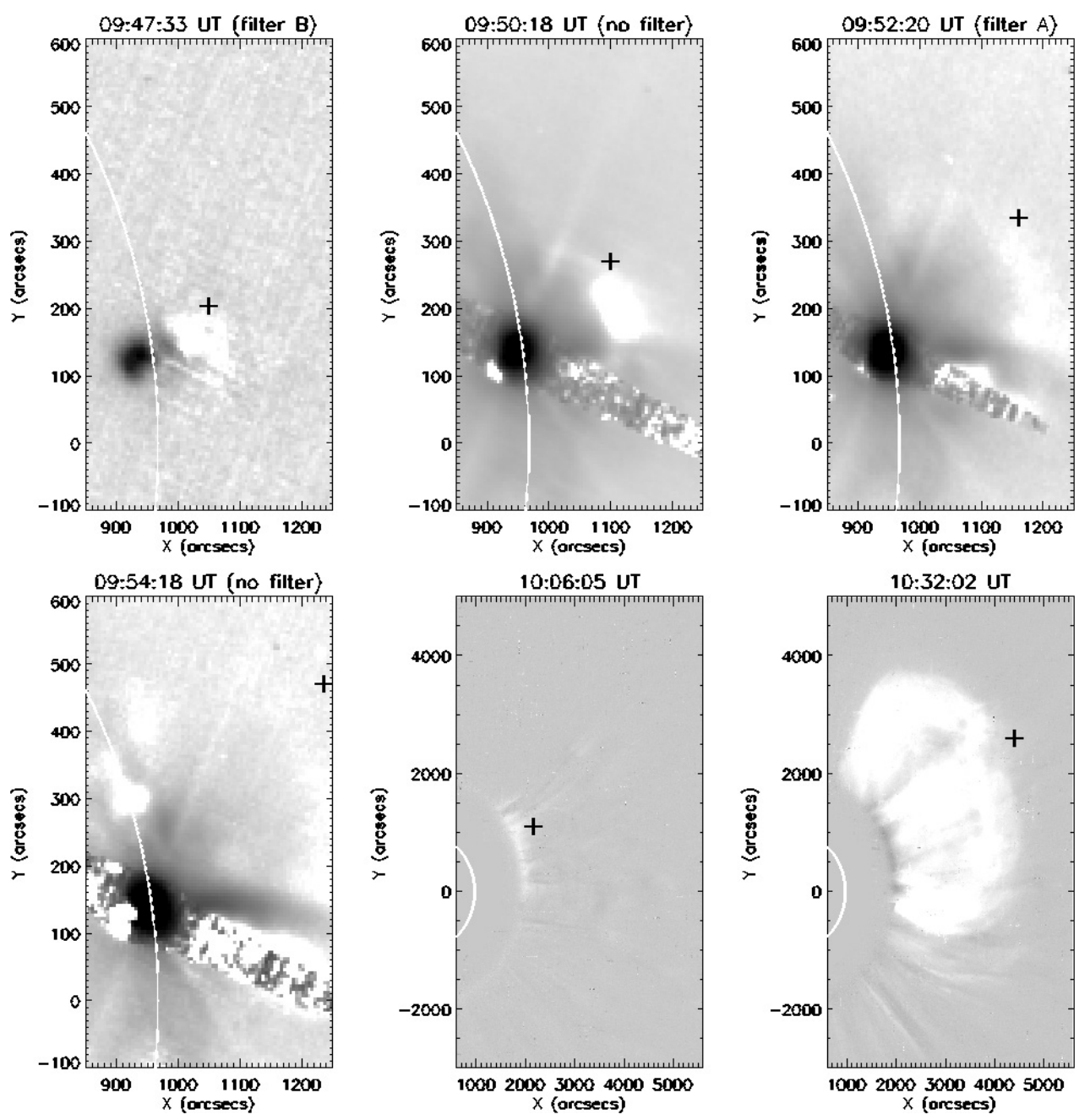

Fig. 3. Panels 1-4: GOES/SXI difference images (reverse colours) showing the evolution and expansion of the loop-like structure. Difference images are built subtracting images taken with the same filter and at the following times: 09:39:33 UT-09:47:33 UT; 10:02:17 UT-09:50:18 UT; 10:00:19 UT-09:52:20 UT; 10:02:17 UT-09:54:18 UT; The X-ray rising loop appears in white in reverse colors as the image showing the expanding loop-like structure is subtracted to an image when this structure is not observed (see text for details). Note the saturation of the detector from the flare in the active region at 09:50:18 UT, 09:52:20 UT and 09:54:18 UT. Panels 5-6: LASCO C2 difference images 10:06:05 UT-09:54:05 UT, 10:32:02 UT-10:06:05 UT showing the CME appearance and expansion. The white curve indicates the solar limb. The crosses indicate the positions at the front of the X-ray expanding loop and of the CME taken to build Figs. 9 and 10.

the acceleration time profile found here seems to be similar to the acceleration time profile determined by e.g. Gallagher et al. (2003) even if the uncertainties on the acceleration values are larger here. An almost constant high acceleration rate is first observed during the impulsive phase of the flare as deduced from the GOES/SXI data points. Later in time, assuming that the Xray and LASCO fronts are closely related (see above) a strong decrease of the acceleration is observed. It is worthwhile noting that the values found in Fig. 5 by the quadratic interpolation of the evolution with time of the heights of the combined X-ray and CME fronts are in good consistency with the velocity and acceleration rates deduced when considering only the first LASCO data points: i.e. velocity of $1400 \mathrm{~km} \mathrm{~s}^{-1}$ and acceleration rate of $37 \mathrm{~m} \mathrm{~s}^{-2}$. Assuming that the X-ray and CME fronts are closely related, no clear indication of the CME acceleration is found at the time corresponding to the re-enhancement of the hard X-ray flux at 09:57:00 UT.

The linear fit of the first values of the velocity deduced from GOES/SXI observations is used to determine the starting time of the X-ray rising loop and CME-proxy. The velocity of the X-ray loop starts to increase from 0 around 09:47 UT which is close to the time of the first GOES/SXI image in which the hot material is seen. This time taken as the launch time of the X-ray rising loop, corresponds to the increase of the radio decimetric/metric fluxes and X-ray flux in the $12-25 \mathrm{keV}$ range. It also corresponds to the first detection of the X-ray source seen by RHESSI (Veronig et al. 2006).

\subsection{Relationship between $X$-ray rising loops, $C M E$ and metric/decimetric radio emissions}

Figure 4 (right panel) shows the spatial extension of the radio sources observed at several frequencies at the time of the continuum emission produced by gyrosynchrotron emission of energetic electrons. The lateral expansion would correspond to the GOES/SXI rising loop if seen at the same altitude. Such an observation is consistent with previous results (see e.g. Maia et al. 1999) who have reported a similar lateral expansion of radio sources corresponding to the CME one.

Thin marks in Fig. 5 shows the time evolution of the projected height and velocity of the front of the combined X-ray rising source and $\mathrm{CME}$ interpolated by a quadratic function and 

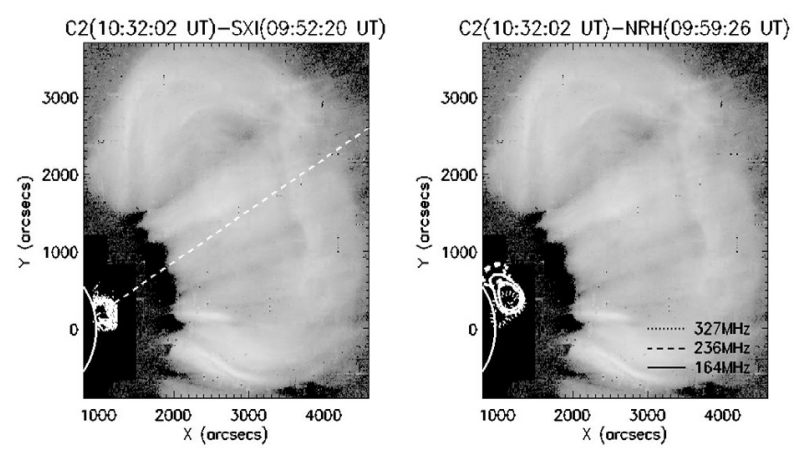

Fig. 4. Left panel: contours of the GOES/SXI difference image at 09:52:20 UT overlaid on the CME observed by LASCO C2 at 10:32:02 UT (difference image). The dashed line shows the axis of the $\mathrm{CME}$. The projected heights of the rising loop and of the CME have been taken along this dashed line. In the right panel: contours at $70 \%$ and $80 \%$ of the radio emissions at $164 \mathrm{MHZ}, 236 \mathrm{MHz}$ and $327 \mathrm{MHz}$ observed at 09:59:26 UT by the NRH overlaid on the CME observed by LASCO C2 at 10:32:02 UT (difference image).

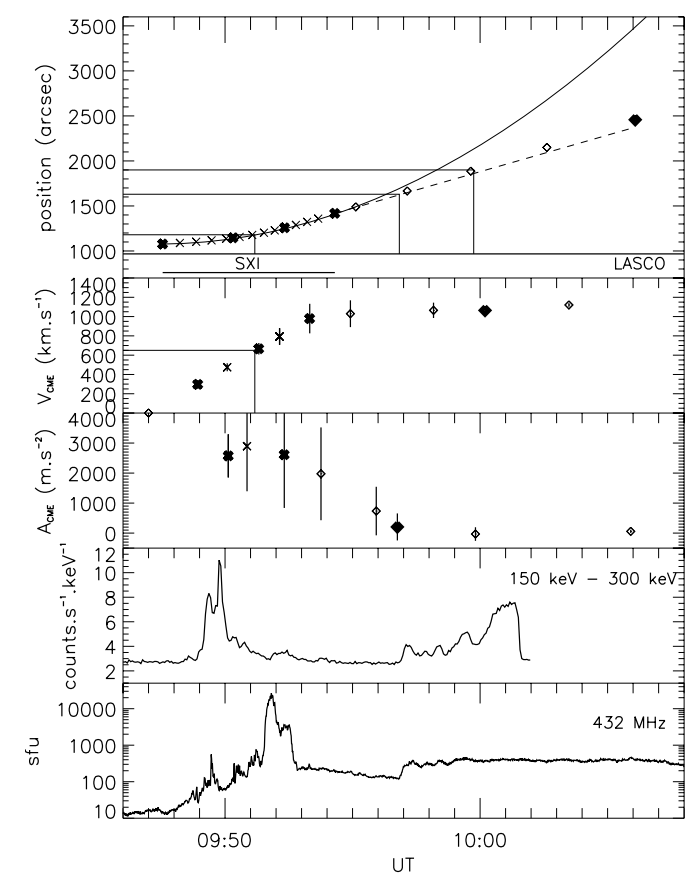

Fig. 5. The three first panels show, respectively, the evolution with time of the projected height, velocity and acceleration of the front of the X-ray rising loop seen with GOES/SXI (thick cross marks) and of the CME seen with LASCO C2 (thick diamond mark) during the 2003 November 03 event. The thin marks represent the quadratic interpolation of the data points. The black curve represents a quadratic fit of the GOES/SXI data points. The dashed line represents the linear extrapolation of the two last GOES/SXI data points. The black lines indicate 09:51:10 UT, 09:57:00 UT and 09:59:45 UT (see text for details). The starting time, indicated by a diamond in the velocity panel is found by linear extrapolation to a null velocity from the two first GOES/SXI data points. The two last panels show, respectively, the time evolution of the RHESSI hard X-ray count rate in the $150-300 \mathrm{keV}$ energy range and of the radio flux at $432 \mathrm{MHz}$.

assuming that they are closely linked. This allows to determine more precisely the velocity and the position of the front of the rising loop at 09:51:10 UT, 09:56:50 UT and 09:59:45 UT, i.e. start of the type II burst at $610 \mathrm{MHz}$, beginning and end of the modulations observed during the second part of the flare. The projected positions of this CME-proxy front are, respectively,

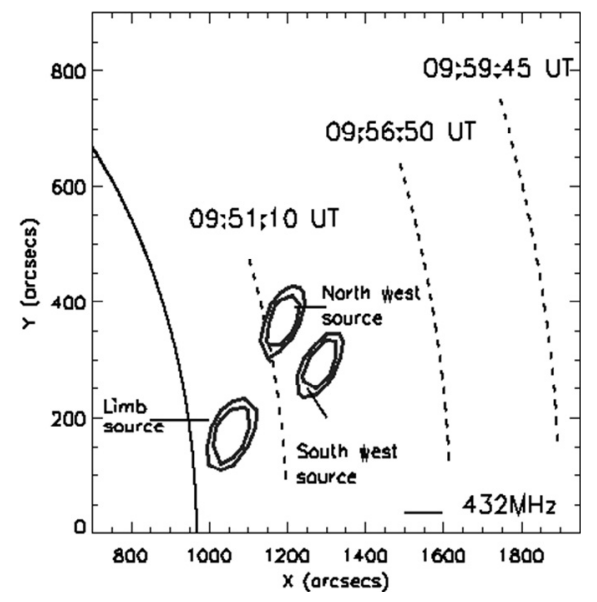

Fig. 6. Compound view of the three main sources at $432 \mathrm{MHz}$ at the time of the emission of the type III-like bursts in the first part of the flare. The NRH contours at $70 \%$ and $80 \%$ shows the limb source at 09:49:18 UT, the North West source at 09:49:41 UT and the south west source at 09:49:13 UT. The dashed lines represent the position of the projected height of the front of the X-ray loops and CME-proxy at 09:51:10 UT, 09:56:50 UT and 09:59:45 UT (see text for details) interpolating between GOES/SXI and LASCO data points.

1180,1630 and 1900 arcsec and the velocity of the X-ray rising loop at the time of the start of the type II burst is around $650 \mathrm{~km} \mathrm{~s}^{-1}$. Note that in the case of a null acceleration after the last GOES/SXI point, the projected positions of the X-ray rising loops and CME-proxy front would be resp. 1630 and 1860 arcsec at 09:56:50 UT and 09:59:45 UT, i.e. very close to the previous values. In the opposite case of a constant acceleration $a_{0}=2746 \mathrm{~m} \mathrm{~s}^{-1}$ deduced from the last GOES/SXI points, the projected positions would be 1700 and 2120 arcsec at 09:56:50 UT and 09:59:45 UT, i.e. above the positions shown in Fig. 5 for the same times.

Positions of radio decimetric/metric sources can be compared to the position of the front of the X-ray rising loop used as a CME proxy in the different assumptions. Figure 6 represents the position of the three main sources at $432 \mathrm{MHz}$ for the impulsive type III like emissions (at 09:49:18 UT). At that time, the $\mathrm{X}$-ray rising loop potentially associated to the CME is well below the north west and south west sources shown in Fig. 6 from where the radio bursts originate (see Fig. 6). The dashed curves represent the position of the X-ray rising front at 09:51:10 UT and of the extrapolated CME-proxy fronts at 09:56:50 UT and 09:59:45 UT. Several comments can be made by comparing the temporal evolution of the radio flux presented in Fig. 1 to the positions of the X-ray loops and CME-proxy front.

- Type III-like bursts at $\mathrm{dm} / \mathrm{m}$ wavelengths are observed up to the beginning of the type II emission. Thus, types III are emitted as long as the X-ray loops and thus the CME-proxy front are well below the west sources.

- Type II emission starts at $432 \mathrm{MHz}$ when the X-ray rising loop (i.e. the CME-proxy front) arrives close to the position of the west sources.

- The X-ray rising loops and thus inferred CME-proxy front are far above the metric radio sources observed by the NRH at the time of the beginning of the modulations. This is true in all the cases considered for the value of the acceleration of the rising loop after the last GOES/SXI data point. Energetic electrons of a few hundreds keV produced at the start of the modulations at X-ray and radio wavelengths thus cannot be accelerated by the shock of the CME at decimetric/metric 
radio emitting heights but must be accelerated and propagate behind the main part of the CME.

\section{Relationship between X-ray rising loop and onset of type II emission}

The harmonic component of the decimetric/metric type II emission is observed during this flare starting at 09:51:12 UT at an unusual high frequency of $\sim 650 \mathrm{MHz}$. It is clearly observed on the spectrogram down to $200 \mathrm{MHz}$ (see Fig. 1) and thus intersects most of the observing frequencies of the NRH allowing to image the position of the type II sources. While the type II harmonic emission at $610 \mathrm{MHz}$ emission (i.e. close to the type II frequency cut-off) mainly consists of one peak, the harmonic emission presents two successive peaks at the lower frequencies observed with the NRH. This could be attributed to two different shocks or to two different preferential emission regions associated with the propagation of one shock. These two harmonic lanes are also seen on the spectra in Fig. 1. The time separation between these two peaks increases with the decrease of the frequency, i.e. with the propagation of the $\operatorname{shock}(\mathrm{s})$.

Figure 7 shows the positions of the type II sources at $432 \mathrm{MHz}, 410 \mathrm{MHz}, 327 \mathrm{MHz}$ and $236 \mathrm{MHz}$ for the times of the two peaks of the harmonic emission defined in Fig. 1. The figure at the top corresponds to the peak times of the first component of the type II harmonic whereas the two other images correspond to the peak times of the second component of the type II. The radio positions are superimposed to the contour of the X-ray rising loop seen by GOES/SXI at 09:52:20 UT and 09:54:18 UT. The GOES/SXI image at 09:52:20 UT is the closest in time to the peak time of the type II emission at $432 \mathrm{MHZ}$ (i.e. the highest frequency that we can image). This image is in fact taken at a time which is very close to the first peak of the type II emission at $327 \mathrm{MHz}$. On the other hand, the image taken at 09:54:18 UT is close to the second peak of the type II at $236 \mathrm{MHz}$. The radio images of the type II emission are taken at different times depending on the frequency. The type II emission starts above the soft X-ray rising loop. The positions of the type II sources at different frequencies are aligned towards the north-west in a quasi-radial direction consistent with the direction of the expansion of the X-ray rising loop. Nevertheless, a different path is observed for the two components of the type II harmonic. The low frequency sources seem to depart more from radial alignment and to bend more to the observer. Different paths for the different type II branches are often observed for type II emissions (e.g. Nelson \& Robinson 1975). This observation is also in agreement with the conclusion of Aurass et al. (1994) who showed that type II sources observed at the same frequency from different split bands can be produced at different sites.

\section{Discussion}

\subsection{Summary of the data analysis}

The main observational characteristics of the 03 November 2003 event are reported in Table 1 and summarized as follows:

1. The hard X-ray flux observed up to $450 \mathrm{keV}$ shows two main parts separated in time by $7 \mathrm{~min}$. A decimetric/metric type II emission is observed between the two parts of the hard X-ray emission starting at an unusual high frequency of $\sim 650 \mathrm{MHz}$.

2. During the impulsive part of the flare, radio type III like bursts are observed at meter/decimeter wavelengths whereas

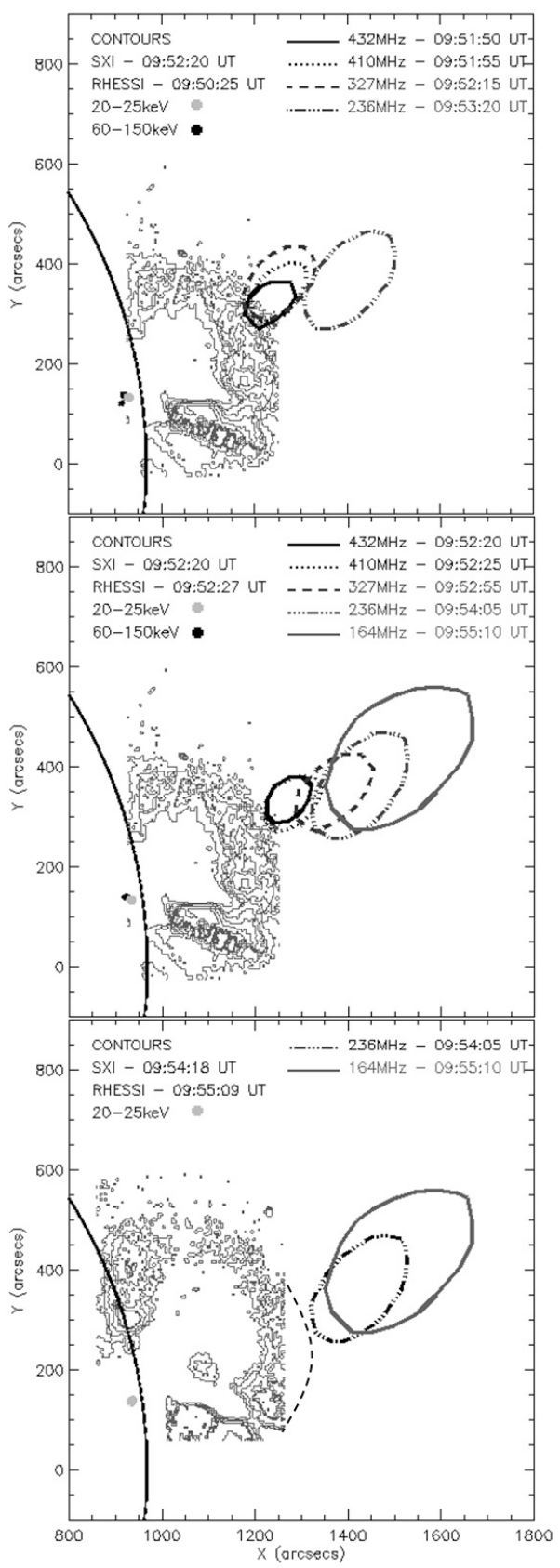

Fig. 7. Contours of the type II sources (taken at $70 \%$ of the maximum) at different frequencies at the times defined in Fig. 1 together with the contours of the GOES/SXI difference images taken, respectively, at 09:52:20 UT and 09:54:18 UT (i.e., the closest to type II peak times). The X-ray sources from RHESSI are imaged in the $20-25 \mathrm{keV}$ (grey) and $60-150 \mathrm{keV}$ (black) energy range. The dashed curve in the latest image shows the extrapolation of the GOES/SXI loop outside of the GOES/SXI field of view.

a strong continuum enhancement with broad-band modulations is observed from $327 \mathrm{MHz}$ to $89 \mathrm{GHz}$ in the second part of the flare.

3. The shape of the radio spectra from $327 \mathrm{MHz}$ to $89 \mathrm{GHz}$ at different times in the second phase of the flare together with the observations of braod-band modulations from the metric to the hard X-ray domains indicate that gyrosynchrotron emission is the prevailing emission mechanism from $327 \mathrm{MHz}$ during the second phase of the flare (see Dauphin et al. 2005). 
Table 1. Time sequence of the main observational characteristics of the 2003 November 3 event.

\begin{tabular}{|c|c|c|c|c|c|c|c|}
\hline $\begin{array}{l}\text { Time } \\
\text { (UT) }\end{array}$ & $09: 45: 00$ & $09: 47: 00$ & 09:49:00 & 09:50-09:52 & $09: 51: 10$ & $09: 56: 45$ & $09: 59: 45$ \\
\hline $\begin{array}{l}\text { Main } \\
\text { charac- } \\
\text { teristics } \\
\text { of X-ray } \\
\text { and radio } \\
\text { emissions }\end{array}$ & $\begin{array}{l}\text { Start of the } \\
\text { soft X-ray } \\
\text { increase } \\
\text { observed } \\
\text { with GOES } \\
(1-8 \AA)\end{array}$ & $\begin{array}{l}\text { Start of } \\
\text { the radio } \\
\text { emission at } \\
432 \text { MHz } \\
\text { (limb } \\
\text { source) } \\
\text { and of the } \\
12-25 \mathrm{keV} \\
\text { X-ray flux. } \\
\text { Launch time } \\
\text { of the X-ray } \\
\text { rising loop }\end{array}$ & $\begin{array}{l}\text { Start of the } \\
\text { first hard } \\
\text { X-ray peak. } \\
\text { Start of } \\
\text { type III - } \\
\text { like emis- } \\
\text { sions at all } \\
\text { frequencies }\end{array}$ & $\begin{array}{l}\text { End of the first } \\
\text { hard X-ray } \\
\text { peak in the } \\
150-300 \text { keV } \\
\text { energy range. } \\
\text { Maximum of } \\
\text { the acceler- } \\
\text { ation of the } \\
\text { X-ray rising } \\
\text { loop }\end{array}$ & $\begin{array}{l}\text { End of the } \\
\text { type III } \\
\text { bursts and } \\
\text { start of } \\
\text { the type II } \\
\text { emission at } \\
432 \text { MHz } \\
\text { in close } \\
\text { temporal } \\
\text { and spatial } \\
\text { relationship } \\
\text { with the } \\
\text { arrival of } \\
\text { the X-ray } \\
\text { rising loop }\end{array}$ & $\begin{array}{l}\text { Start of the } \\
\text { hard X-ray } \\
\text { and radio } \\
\text { modulations. } \\
\text { End of the } \\
\text { type II emis- } \\
\text { sion. The } \\
\text { extrapolated } \\
\text { position of } \\
\text { the CME } \\
\text { front is far } \\
\text { above the } \\
\text { radio sources }\end{array}$ & $\begin{array}{l}\text { End of } \\
\text { the hard } \\
\text { X-ray mod- } \\
\text { ulations } \\
\text { observed } \\
\text { by RHESSI }\end{array}$ \\
\hline
\end{tabular}

4. GOES/SXI observations show expanding rising loops. When extrapolated to the height of the first CME detection these rising loops are consistent with the CME observations in terms of spatial extent, height and velocity.

5. The X-ray rising loop presumably associated to the CME starts to rise at 09:47 UT at the time of the increase of the radio decimetric/metric fluxes and of the X-ray flux in the $12-25 \mathrm{keV}$ range. This is well before the starting time of the CME derived from the height-time analysis of LASCO data.

6. Type III-like bursts are observed up to the beginning of the type II emission as long as the X-ray rising loop is below the west radio sources.

7. A clear temporal and spatial association is observed between the GOES/SXI X-ray rising loop and the onset of the type II burst at the highest frequency which can be imaged by the NRH.

8. The extrapolated positions of the X-ray rising loop and thus of the associated CME front are well above the metric radio sources observed by the NRH at the time of the beginning of the modulations observed in the hard X-ray and radio domains.

\subsection{Flare/CME scenario of the 03 November 2003 event}

\subsubsection{Kinematics of the $X$-ray rising loop and of the CME}

The X-ray rising loop observed by GOES/SXI propagates in a direction and has a spatial extent which can be related to the ones of the CME observed in C2 at 10:06:05 UT and 10:32:02 UT. A quasi constant acceleration of the X-ray loop can reproduce the kinematics of the X-ray feature between 09:47:33 UT and 09:54:18 UT. This acceleration rate around $2700 \mathrm{~m} \mathrm{~s}^{-2}$ is much larger than the one deduced later in the event from the LASCO data points (around $30 \mathrm{~m} \mathrm{~s}^{-2}$ ).

The height of the first CME detection in LASCO C2 at 10:06:08 UT is close to the extrapolated height of the $\mathrm{X}$-ray loop calculated with a null acceleration (i.e. a constant speed $\sim 1000 \mathrm{~km} \mathrm{~s}^{-1}$ after 09:54:18 UT). Though GOES/SXI and $\mathrm{SOHO} / \mathrm{LASCO}$ observations most probably probe different plasma conditions, the morphology of the X-ray rising loops and the comparison of the extrapolated and observed heights suggests a link with the CME observed higher in the corona with LASCO. Assuming such a relationship, the height, velocity and acceleration time profiles of the propagating structure are similar in some extent to the case already observed by Gallagher et al. (2003) who have studied the CME kinematics at low altitudes using TRACE, UVCS and LASCO data. In both cases, the maximum acceleration rate occurs at about the same time or shortly after the maximum of the hard X-ray flux. However the height, velocity and acceleration time profiles are better described in Gallagher et al. (2003) given the higher time cadence of the TRACE observations and contrary to the present observations the acceleration time profile is better described by an exponential rise.It must furthermore be noticed that the present results on the CME kinematics discussed in details in Sect. 3 are in disagreement with those reported by Vrsnak et al. (2006) who claim a maximum acceleration of the GOES/SXI-CME structure between 09:55 UT and 10:00 UT. They however do not show the details of their analysis. Given the spatial extent of the rising loop and the intrinsic 3D structure of the phenomenon, it is not impossible that different parts of the soft X-ray loop may exhibit different height-time profiles, once projected on the plane of sky. The present analysis is performed in the direction of the type II source and the deduced height-time, velocity and acceleration profiles are therefore appropriate for the comparison of the type II onset at high frequencies.

Finally, the present analysis of the propagation of the X-ray loop and CME front combining GOES/SXI and LASCO observations supports the scenario of the kinematics of the CME evolution described by Zhang et al. (2001) based on combined observations of LASCO C1, C2 and C3. They indeed propose a three-phase evolution scenario. The initiation phase, starting in the present event with the radio decimetric/metric flux and $12-25 \mathrm{keV} \mathrm{X}$-ray flux increases, corresponds to the slow rise phase. The following phase is the impulsive acceleration phase in which the acceleration reaches its maximum value slightly after the hard X-ray peak flux time. A quasi constant velocity found here around $1200 \mathrm{~km} \mathrm{~s}^{-1}$ characterizes the last phase observed after 09:55 UT (i.e. the propagation phase). All these similarities with the observations from Zhang et al. (2001) strongly suggest that the X-ray rising loop observed in GOES/SXI can characterize the evolution of the CME emission at low altitude in the corona.

The launch time of the X-ray rising loop then believed to correspond to the CME one is found to be around 09:47:00 UT in close connection with the start of the flare in the decimet$\mathrm{ric} / \mathrm{metric}$ and $12-25 \mathrm{keV}$ ranges. This is earlier than the time given from a simple extrapolation of LASCO data and one 
minute later than the one given in Vrsnak et al. (2006). The basic scenario which may be envisaged would be related to some primary energy release leading to first flare signatures. In close connection with this energy release, hot X-ray loops start to expand as observed with GOES/SXI.

Final comments can be made on the comparison between the location of the "SXI global coronal wave" studied by Warmuth et al. (2005), the results of Vrsnak et al. (2005) and our observations. In their paper, Warmuth et al. (2005) have reported first observations of a global GOES/SXI wave propagating to the North and related to an EIT and Moreton waves already reported by Vrsnak et al. (2005). They interpreted the time evolution of the different waves as the signature of a single physical disturbance. A polynomial fit of the different front wave positions indicates that the wave seems to start around the impulsive phase of the flare but the lack of observations of the waves close to the active region does not allow an exact evaluation of the wave starting time. The rising loop that we report here may also be related to the waves. Indeed, the GOES/SXI observations shown in Fig. 4 also reveal an extension of the X-ray loop like features towards the North. This could correspond to a separation of the location where these X-ray features are anchored at the solar surface and to a movement to the north of the northern anchor point. This movement could be connected to the propagation to the north of the global wave reported by Warmuth et al. (2005).

\subsubsection{X-ray rising loop and type II emission}

The position of the type II burst at its onset compared to the position of the X-ray rising loop at a close time strongly suggests that the origin of the type II burst is closely associated to the dynamics of the X-ray rising loop. The type II radio source with a peak time the closest to the time of the X-ray image (i.e. $327 \mathrm{MHz}$ at 09:52:20 UT and $236 \mathrm{MHz}$ at 09:54:18 UT) is just ahead of the X-ray disturbance. This suggests that the propagation of the rising loop creates a shock front ahead of the loop when the propagation velocity becomes larger than the local Alfvén speed thus leading to type II emission. From our kinematics analysis of the $\mathrm{X}$-ray rising loops, its velocity is more than $650 \mathrm{~km} \mathrm{~s}^{-1}$ at the start time of the type II burst. The radiations created at the shock wave are emitted at the harmonic local plasma frequency which is also the emission frequency of the type III bursts. It is thus expected that the type II emission begins at a position close to the type III sources at high frequencies, which is observed here.

As mentioned in the introduction, association between X-ray ejecta and onset of high frequency type II emission has been already observed (e.g. Klein et al. 1999; Gopalswamy et al. 1997). However, this study was based on YOHKOH/SXT observations probing hotter plasmas than the ones observed with GOES/SXI. This is here the first observation with GOES/SXI of a rising Xray loop associated with the onset of a high frequency type II emission. The timing and the location of the type II with respect to the rising X-ray loops suggest that the driver of the type II burst is the propagation of the coronal structure observed in the $\mathrm{X}$-ray wavelength by GOES/SXI.

The type II sources are not observed along the whole front of the X-ray loop but are produced along limited paths. As emphasized in Steinolfson (1984), several parameters, such as the shock velocity or the angle between the normal to the shock and the ambient magnetic field can play a role in the location of the type II sources. In the present case, the type II sources are located at the top and close to the direction of expansion of the X-ray rising loops. The type II sources are also close to the locations of the type III sources at the same frequency which indicated open magnetic field lines. These combined observations could indicate electron acceleration in a quasi parallel situation. These preferential emission regions could also correspond to the intersection of the shock wave with pre-existing magnetic structures (e.g. Stewart 1984). It can finally be noticed that a mechanism for accelerating electrons to suprathermal energies at quasiparallel shock waves in the corona has been investigated by e.g. Mann \& Classen (1995).

The time evolution of the harmonic type II burst from $432 \mathrm{MHz}$ to $236 \mathrm{MHz}$ shows two successive peaks which could be attributed to two different shock waves or to two different preferential emission regions associated with the propagation of one shock. The highest frequency sources of the first branch show a quasi radial propagation whereas the second branch seems to propagate more in the sun earth direction. These two branches may thus have a different angle between the direction of the density gradient and the normal to the shock leading to different peak times and frequency drifts.

It is finally worthwhile noting that the shock wave driven by the rising loop accelerates only electrons which produce the type II emission but do not produce efficiently hard X-ray emitting electrons. This has been discussed in more details by Klein et al. (2003) and most probably shows that the number of high energy electrons accelerated by the shock is in fact too low to allow the production of X-ray radiation by an incoherent process.

The paper of Vrsnak et al. (2006) rather favor a flare-ignited wave scenario for the production of the coronal shock at the origin of the Moreton wave and the associated type II burst emission. One of their arguments is based on the comparison between the velocity of the GOES/SXI loop at the type II onset and the velocity of the shocks inferred from the radio spectra between 09:52 UT and 09:55 UT. They conclude that the velocity of the GOES/SXI-CME leading edge is considerably smaller that the velocity of the shock at the time of the type II burst. However, while the shock velocities derived in their analysis from the dynamic spectrum and a coronal density model are respectively $1800 \mathrm{~km} \mathrm{~s}^{-1}$ and $1100 \mathrm{~km} \mathrm{~s}^{-1}$, the direct determination of the projected shock velocities from the radio images lead to $1300 \mathrm{~km} \mathrm{~s}^{-1}$ for the first branch and $1100 \mathrm{~km} \mathrm{~s}^{-1}$ for the second branch, thus reducing the discrepancies between shock and GOES/SXI-CME velocities. Furthermore, the present analysis shows that between 0952 and 0955 UT (time interval used to derive the shock speed), the GOES/SXI rising loop has a velocity which increases from $650 \mathrm{~km} \mathrm{~s}^{-1}$ to $1000 \mathrm{~km} \mathrm{~s}^{-1}$ so that the speed of the rising loop derived from our analysis at 09:55 UT is not inconsistent with the velocity of the type II burst sources. This is the reason why the direct comparisons of radio images of the type II burst and X-ray images from GOES/SXI shown in Fig. 7 show such a good agreement. Based on our data analysis, it can thus be concluded that in the present case, the CME associated X-ray rising loop impulsively launched at low altitudes is the main cause of the type II burst which starts here at an unusual high frequency in the decimeter wavelength range.

\subsubsection{Enhancement of the radio and hard X-ray emissions after 09:57:00 UT}

After 09:57:00 UT, a new enhancement of the hard X-ray flux above $100 \mathrm{keV}$ is observed in association with a strong continuum emission in the whole range from several tens of $\mathrm{GHz}$ to almost $200 \mathrm{MHz}$ (see Dauphin et al. 2005). Moreover, the time evolution of the hard X-ray and radio flux in the whole frequency band presents several modulations in phase in the radio and X-ray domains indicating that the acceleration of X-ray and 
radio emitting electrons is closely linked and potentially arise from a same region. No motion of the radio sources is observed in this phase in our case. As shown in Dauphin et al. (2005) gyrosynchrotron emission is the prevailing emission mechanism for the broad-band emission even at decimetric wavelengths and this emission probably originates from electrons trapped in closed magnetic field structures (Dauphin et al. 2005).

The extrapolated positions of the X-ray rising loop and thus of the CME-proxy front are well above the metric radio sources observed by the NRH at the time of the beginning of the hard $\mathrm{X}$-ray and radio modulations. The energetic electrons produced at the time of the modulations thus cannot be accelerated by the shock of the CME but must be accelerated and propagate behind the main part of the CME.

A possible acceleration site for these electrons would be the reconnection sites which can be triggered in the current sheets behind erupting flux ropes, for example through tearing mode instability (e.g. Odstrcil \& Karlicky 1997). These instabilities could also be a possible explanation of the modulations observed at the beginning of the second phase. Indeed, tearing mode (Furth 1964) is a time dependent process which invokes the development of a series of magnetic islands with corresponding X-type and O-type neutral lines.

\section{Conclusions}

This paper presents the detailed observations of an X-ray rising loop associated to the onset of a type II burst at high frequencies for the 03 November 2003 flare. The present analysis is focussed on the detailed kinematics of the GOES/SXI loop front in order to understand the relationship with the type II burst onset. The analysis shows that the GOES/SXI rising loop can be related to the signature at low altitude of a CME observed in LASCO C2. The velocity and acceleration time profiles found for the rising loop and the associated CME are consistent with an impulsive acceleration at low altitudes which has already been found for other events. The fast acceleration of the coronal loop after its launch leads at the time of the onset of the type II emission to a velocity of the loop of the order of the decimetric/metric type II velocity derived from the radio images. Moreover, the direct comparison of the type II sources with the GOES/SXI observations clearly shows that the type II burst is ignited by the shock wave created ahead of the rising X-ray loop. The present event then shows that CME associated X-ray loops impulsively launched from very low heights may cause high frequency type II bursts, starting in the decimetric wavelength range.

The production of energetic electrons in the second acceleration phase observed during this flare is the other point studied in this paper. Indeed, the peculiarity of this event is the presence of two phases of intense emissions from energetic electrons separated in time by a few minutes. The second phase of the flare, observed after the type II emission, is characterized by a new increase and modulations of the hard X-ray and radio flux from $300 \mathrm{MHz}$ to several tens of $\mathrm{GHz}$. Modulations of braodband radio emissions in close time coincidence with hard X-ray modulations have been reported for other flares (see e.g. Asai et al. 2001; Pick et al. 2005). As suggested in Pick et al. (2005), these broad-band modulations are interpreted in the framework of an erupting flux rope (even if there is no direct evidence of the twisted flux rope) with the formation of a current sheet behind. In this scenario, particle acceleration occurs at the reconnection sites triggered in the current sheets. Modulations with a period around $40 \mathrm{~s}$ at the beginning of the second phase of the flare could be an indication of MHD instabilities in the tail of the flux rope. As a conclusion, while the type II burst is the signature of the shock wave created ahead of an ascending flux rope, the long duration emission will be due to the reconnection in the tail of the flux rope. At higher altitude, a CME associated with the erupting flux rope is finally observed.

Events showing such a second phase are rare. Similar events could be searched for in the RHESSI data. The analysis of the hard X-ray, radio flux time evolution combined with observations of mass motions at low altitude could provide contraints on the conditions needed to produce such events.

Acknowledgements. Cyril Dauphin and Nicole Vilmer acknowledge support from the Centre National d'Etudes Spatiales on the RHESSI project. The Nançay Radio Observatory is funded by the French Ministry of Education, the CNES and the Région Centre. The authors acknowledge useful comments from the referee.

\section{References}

Alexander, D., Metcalf, T., \& Nitta, N. V. 2002, GRL, 29, 41

Aurass, H., Klein, K. L., \& Mann, G. 1994, in Solar Dynamics Phenomena and Solar Wind Consequences, ed. J.J. Hunt, ESA SP-373, 95

Asia, A., Shimojo, H., Isobe, H., et al. 2001, ApJ, 562, L103

Brueckner, G. E., Howard, R. A., Koomen, M. J., et al. 1995, Sol. Phys., 162, 357

Cliver, E. W., Nitta, N. V., Thompson, B. J., \& Zhang, J. 2005, Sol. Phys., 225, 105

Cremades, H., \& Bothmer, V. 2004, A\&A, 422, 307

Dauphin, C., Vilmer, N., Luthi, T., et al. 2005, AdSPR, 35, 10, 1805

Delaboudiniere, J.-P., Artzner, G. E., Brunaud, J., et al. 1995, Sol. Phys., 162, 291

Furth, H. P. 1964, in Advanced Plasma Theory, ed. M. N. Rosenbluth (New York: Academic Press), 159

Gallagher, J. S., Madsen, G. J., Reynolds, R. J., et al. 2003, ApJ, 588, 326 Gopalswamy, N., Kundu, M. R., Manoharan, P. K., et al. 1997, ApJ, 486, 1036 Gopalswamy, N., Kaiser, M. L., Sato, J., \& Pick, M. 2000, ASPC, 206, 351 Gopalswamy, N., Yashiro, S., Kaiser, M. L., Howard, R. A., et al. 2001, JGR, 106, A12, 29219

Handy, B. N., Acton, L. W., Kankelborg, C. C., et al. 1999, Sol. Phys., 187, 229 Hill, S. M., Pizzo, V. J., Balch, C. C., et al. 2005, Sol. Phys., 226, 255

Hudson, H. S., Acton, L. W., \& Freeland, S. L. 1996, ApJ, 470, 629

Hudson, H. S., Khan, J. I., Lemen, J., et al. 2003, Sol. Phys., 212, 121

Kerdraon, A., \& Delouis, J. M. 1997, in Coronal Physics from Radio and Space

Observations, ed. G. Trottet (Heidelberg, Germany: Springer-Verlag), 192 Khan, J. I., \& Aurass, H. 2002, A\&A, 383, 1018

Klassen, A., Aurass, H., Klein, K. L., et al. 1999, A\&A, 343, 287

Klassen, A., Aurass, H., Mann, G., \& Thompson, B. J. 2000, A\&AS, 141, 357

Klein, K. L., Khan, J. I., Vilmer, N., et al. 1999, A\&A, 346, L53

Klein, K. L., Schwartz, R. A., McTiernan, J. M., et al. 2003, A\&A, 409, 317

Kohl, J. L., Esser, R., Habbal, S., et al. 1995, Sol. Phys., 162, 313

Lin, R. P., \& the RHESSI team 2002, Sol. Phys., 210, 3

Liu, W., Jiang, Y. W., Liu, S., \& Petrosian, V. 2004, ApJ, 611, L53

Maia, D., Vourlidas, A., Pick, M., et al. 1999, JGR, 104, A6, 12507

Mancuso, S., \& Raymond, J. C. 2004, A\&A, 413, 363

Mann, G., \& Classen, H.T. 1995, A\&A, 304, 576

Maxwell, A., Dryer, M., \& McIntosh, P. 1985, Sol. Phys., 97, 401

Moreton, G. E. 1960, AJ, 65, 494

Nelson, G. J., \& Robinson, R. D. 1975, Proc. of the Astronomical Society of Australia, 2, 370

Nelson, G. J., \& Melrose, D. R. 1985, in Solar Radiophysics, ed. D. J. McLean, \& N. R. Labrum (Cambridge, UK: Cambridge Univ. Press), 333 Odstrcil, D., \& Karlicky, M. 1997, A\&A, 326, 1252

Pick, M., Demoulin, P., Krucker, S., et al. 2005, ApJ, 625, 1019

Reiner, M. J., Kaiser, M. L., \& Bougeret, J.-L. 2001, JGR, 10629989R

Steinolfson, R. S. 1984, Sol. Phys., 94, 193

Stewart, R. T. 1984, Sol. Phys., 94, 379

Thompson, B. J., Plunckett, S. P., Gurman, J. B., et al. 1998, GRL, 25, 2461

Uchida, Y. 1960, Publications of the Astronomical Society of Japan, 12, 376

Uchida, Y. 1974, Sol. Phys., 39, 431

Veronig, A. M., Karlicky, M., Vrsnak, B., et al. 2006, A\&A, 446, 675

Vilmer, N., Pick, M., Schwenn, R., et al. 2003, Annales Geophysicae, 21, 847

Vrsnak, B., \& Lulic, S. 2000, Sol. Phys., 196, 181

Vrsnak, B., Magdalenic, J., Temmer, M., et al. 2005, ApJ, 625, L67

Vrsnak, B., Warmuth, A., Temmer, M., et al. 2006, A\&A, 448, 739

Warmuth, A., Mann, G., \& Aurass, H., et al. 2005, ApJ, 626, L121

Wild, J. P., 1962, J. Phys. Soc. Japan, 17, 249

Zhang, J., Kundu, M. R., White, S. M., et al. 2001, ApJ, 559, 452 